\title{
Escolher a especialidade de medicina geral e familiar Opção inicial ou uma alternativa?
}

Dina Gaspar*

\section{RESUMO}

Objectivos: Avaliar o grau de influência dos factores identificados para a escolha da especialidade de Medicina Geral e Familiar, em Portugal; analisar a importância de factores intrínsecos à especialidade, nas escolhas profissionais da referida população. Tipo de Estudo: Estudo observacional, transversal e analítico.

Local: Portugal continental.

População: Médicos que iniciaram o Internato Médico de Medicina Geral e Familiar, durante o ano de 2005 (N = 228 ).

Métodos: Inquérito por questionário administrado a todos os médicos, solicitando que indicassem a importância de um conjunto de factores na sua escolha da especialidade (14 itens numa escala tipo Likert de 5 pontos). Determinação de estatísticas descritivas e análise bivariada, para comparação de médias em função da caracterização da população $(\alpha=0,05)$.

Resultados: Do total de médicos, $109(47,8 \%)$ responderam ao questionário. Os motivos intrinsecamente relacionados com as características da especialidade $(M=26,9 ; D P=4,24)$ foram mais relevantes, contrariamente aos motivos de ordem pessoal ou outros $(M=17,4 ; D P=4,13)$, na opção pela Medicina Geral e Familiar. A sensibilização para a Medicina Geral e Familiar durante a licenciatura é referida como tendo alguma influência $(M o=3)$ por $23,4 \%$ dos médicos.

Conclusões: Para a maioria dos médicos que iniciam o Internato Médico de Medicina Geral e Familiar, a opção por esta especialidade parece ser actualmente congruente com motivos intrinsecamente relacionados com as características da mesma. Considera-se a necessidade de repensar o papel da disciplina de Medicina Geral e Familiar no currículo médico pré-graduado e a influência da escola médica na opção por esta especialidade.

Palavras-chave: Medicina Geral e Familiar; Internato médico; Motivos Invocados para Escolher uma Especialidade; Escola Médica.

\section{INTRODUÇÃO}

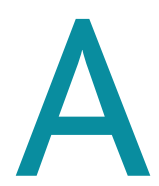

nível internacional são actualmente propostos vários cenários de mudança dos paradigmas da Medicina Geral e Familiar (MGF), perante a já conhecida crise dos Cuidados de Saúde Primários. ${ }^{1}$ Para este fenómeno contribui o declínio de interesse dos médicos por especialidades generalistas, entre as quais a MGF. ${ }^{2-7}$

Em Portugal, vários autores têm vindo a debruçar-se sobre esta problemática, apontando para possíveis causas implicadas na redução da taxa de ocupação de vagas do internato médico de $\mathrm{MGF}{ }^{8-10}$ relacionada com a presumível ausência de interesse dos estudantes de medicina e jovens médicos pela mesma. Dados da úni-

*Assistente Graduada de Medicina Geral e Familiar. Mestre em Educação Médica. Centro de Saúde de Faro - Administração Regional de Saúde do Algarve ca investigação publicada em Portugal sobre esta temática, ${ }^{11}$ realizada na zona Norte do país em 2000 , revelam que os motivos relacionados com as características desta especialidade foram referidos em percentagem mais elevada (75\%), sobrepondo-se aos motivos relacionados com expectativas de ordem pessoal (56\%), como a disponibilidade para a vida familiar, que parecem ter menor importância na escolha de MGF. Para os que não escolheram MGF como primeira opção, a nota de acesso foi a condicionante da escolha e a remuneração não parece ter sido um motivo importante com influência nas escolhas de MGF, para o grupo estudado.

Estudos de revisão sobre factores associados a escolhas de carreira em jovens médicos graduados em países europeus ${ }^{12}$ identificam factores pessoais e factores relacionados com as características da especialidade, 
para além da influência de experiências anteriores. O interesse humanístico (72\%), a motivação (62-68\%) e a auto-satisfação relacionada com expectativas profissionais (52-68\%) são os factores identificados como as principais características pessoais associadas à escolha de uma especialidade..$^{12}$ As circunstâncias relacionadas com a vida pessoal têm uma associação menor (18$-40 \%$ ) e são mais importantes para as mulheres, assim como para os médicos que escolhem Medicina Familiar, do que para os médicos que escolhem o hospital. Expectativas em relação às condições de trabalho (41$-48 \%$ ), horas de trabalho (44-47\%), aspectos de promoção de carreira (27-58\%) e aspectos financeiros (12$-49 \%)$ são as principais características relacionadas com a profissão que influenciam as escolhas. ${ }^{12}$

As mulheres parecem ter maior preferência pela MGF, mais compatível com a vida familiar do que uma especialidade hospitalar, ${ }^{12}$ para além de estar descrito uma maior apetência das mulheres por especialidades em que é maior a componente da compaixão, do contacto com o doente e das relações interpessoais. ${ }^{13}$

Os factores com forte associação à escolha de MGF pelos jovens médicos recém-licenciados ${ }^{14}$ são aqueles relacionados com maior orientação social (centrada no envolvimento social e intervenção comunitária, assim como na relação médico-doente mantida no tempo), e com uma prática clínica mais abrangente (diversidade de pacientes e de problemas de saúde). Parece haver, na opção pela MGF, uma maior relação com a idade mais avançada dos médicos e uma maior preocupação com os estilos de vida profissional. ${ }^{14}$

As preferências por uma especialidade à entrada da faculdade são um factor determinante, mas as características da faculdade de origem e a influência dos seus Departamentos de Clínica Geral, parecem ter tido pouca importância na preferência pela MGF ${ }^{14}$ Contudo, o mesmo estudo revela que faculdades que têm um programa longitudinal, com forte componente da Medicina Geral e Familiar, parecem influenciar o número de estudantes que escolhe esta especialidade. Permitem o desenvolvimento de uma cultura informal que é propícia à MGF, através de opiniões, comentários, e atitudes positivas por parte dos professores, tutores e estudantes, com efeitos positivos na opção dos jovens médicos pela MGF. ${ }^{14}$

Sabe-se igualmente que a influência de excelentes professores e a exposição ao papel de «modelos» ${ }^{15}$ parece ser um importante preditor de escolha para excelentes alunos no sentido de uma especialidade, podendo no entanto ter influências tanto positivas como negativas nessas escolhas..$^{14} \mathrm{~A}$ existência de figuras de referência no contexto do ensino pré-graduado e treino pós-graduado poderá influenciar a escolha da especialidade.

Ao preferirem uma especialidade com as características da MGF os estudantes de medicina pretendem ter um trabalho diferente do trabalho hospitalar, «mais próximo das pessoas», dos problemas sociais e comunitários, para além dos factores de ordem pessoal já referidos. A percepção de um largo espectro de actuação ou abrangência da sua prática pode ser igualmente um factor importante na decisão, em especial quando os estudantes são mais acompanhados por tutores de MGF. ${ }^{16}$

Podemos concluir que as variáveis positivamente correlacionadas com as escolhas de carreira em Cuidados de Saúde Primários incluem os valores humanísticos, e dizem respeito a «trabalhar com pessoas»e à «situação familiar» dos médicos, sendo a «remuneração e o prestígio» os factores referidos com correlação negativa. ${ }^{17}$

Estudos publicados mais recentemente em Portugal permitem concluir que se verifica uma evolução positiva no padrão de escolha por esta especialidade ${ }^{18}$ sendo mais elevada a percentagem de médicos que se decidem pela MGF como a sua primeira opção, contrariando a opinião geral de que os médicos que a escolhem fazem-no por ausência de alternativas e não como uma prioridade para o seu futuro profissional. Por outro lado, o aparente aumento da classificação média na prova nacional de seriação dos médicos que escolhem MGF, nos últimos cinco anos, parece-nos reforçar a percepção de uma inversão positiva do padrão de preferências dos jovens médicos, à entrada da especialidade.

Considerando-se incipiente a investigação realizada sobre esta matéria, neste trabalho procuramos abordar a problemática das razões que levam os médicos a escolher MGF, no actual contexto social e político, em que se assiste a mudanças substanciais no âmbito da reforma dos Cuidados de Saúde Primários.

Estarão os médicos que optam por MGF realmente 
motivados para esta especialidade, escolhendo esta opção como reflexo de um interesse particular pelas características inerentes ao exercício da mesma? Ou será que estes médicos escolhem MGF como uma alternativa, face à nota insuficiente e consequente dificuldade de acesso a especialidades hospitalares? Será a classificação de candidatura, uma condicionante, restando a estes médicos a possibilidade de experimentar a MGF, enquanto aguardam por outras alternativas? Ou estarão os médicos a optar por uma especialidade que supostamente lhe proporcionaria maior estabilidade profissional e mais tempo livre para a família e vida privada? Estarão os médicos que iniciam o Internato Médico de Medicina Geral e Familiar (IMMGF) a atribuir maior importância a aspectos relacionados com interesses pessoais e circunstâncias de vida, quer pessoal quer profissional? Até que ponto é que os aspectos extrínsecos ao exercício da própria especialidade, nomeadamente a possibilidade de ter uma boa remuneração, terão importância substancial neste grupo profissional? Terão as experiências profissionais anteriores, ou seja, o contacto com o exercício da especialidade de MGF, incluindo a experiência adquirida durante os estágios de MGF, nomeadamente no contexto da licenciatura e no Internato Geral, influenciado de forma consistente a escolha? Terá a escola médica influenciado o percurso dos médicos no sentido de uma maior sensibilização para esta especialidade?

Com a finalidade de analisar a problemática das escolhas profissionais dos médicos que optam pela especialidade de MGF, no início da sua formação em internato médico, contribuindo igualmente para consolidar a informação já existente sobre esta matéria no nosso país, foram definidos os seguintes objectivos para a presente investigação:

- Avaliar a influência dos factores invocados para a escolha da especialidade de Medicina Geral e Familiar na população de médicos internos que optam por esta especialidade;

- Determinar a importância de motivos intrinsecamente relacionados com as características da especialidade na opção pela Medicina Geral e Familiar, na referida população.

\section{MÉTODOS}

A metodologia utilizada, anteriormente reportada pela autora, ${ }^{18}$ incluiu a aplicação de um questionário aos médicos que optaram pela especialidade de MGF em Portugal continental durante o ano de $2005(\mathrm{~N}=228)$. O questionário incluía um conjunto de 14 itens, representando razões invocadas para escolher MGF, valorizados pelos respondentes, segundo uma escala tipo $L i$ kert, com cinco opções de resposta. A resposta aos itens da escala varia na sua pontuação desde o valor $1=$ «nenhuma» até ao $5=$ «muitíssima», relativamente ao grau de influência que cada um dos motivos contribuiu para as escolhas de MGF por estes médicos.

Para identificar os «Motivos de Escolha da especialidade de MGF» procedeu-se à formulação de um conjunto de itens, utilizando a seguinte metodologia:

- Selecção e listagem dos motivos de escolha, identificados a partir da literatura, ${ }^{3,6,11-12,14,17,19}$ assim como através de entrevistas individuais realizadas a um grupo de médicos com as mesmas características da população em estudo;

- Estudo preliminar do instrumento que contém os itens representando os motivos de escolha, através de uma metodologia de «reflexão falada $»^{20}$ junto de um grupo de médicos de MGF, no sentido da identificação de informação relevante acerca do domínio que se pretende conhecer e concluir pela selecção dos 14 itens, considerados para a construção da versão final;

- Validação por um júri de especialistas, para apreciação da compreensibilidade das instruções e dos itens, tendo em conta os objectivos do estudo e as características da população alvo. ${ }^{20}$

Depois de seleccionados, os motivos de escolha de MGF foram agrupados em duas categorias - Factores Intrínsecos e Factores Extrínsecos, identificando os motivos atribuíveis a aspectos intrinsecamente relacionados com a especialidade de MGF (I) e os motivos relacionados com factores extrínsecos à mesma (E), ou seja, atribuíveis a interesses pessoais, estilos de vida, influência social e dificuldades de acesso à licenciatura em Medicina.

- Variáveis medidas:

- Instrumento de medida, contendo um conjunto de itens que identificam a dimensão «Motivos de escolha da especialidade de MGF», classificados em extrínsecos (E) e intrínsecos (I): garantia de maior estabilidade profissional (E); gostar de aju- 
dar pessoas doentes (I); ter maior autonomia e independência profissional (E); contacto anterior com o exercício da especialidade de MGF, em estágios em Centros de Saúde (I); ter maior disponibilidade para família e outros interesses, não profissionais (E); maior abrangência de áreas médicas abordadas (I); prestígio social da MGF (E); sensibilização durante a licenciatura para a disciplina de MGF (I); possibilidade de ter uma boa remuneração (E); gostar do contacto com «pessoas» (I); «nota de classificação baixa» no exame de ingresso à especialidade (E); vocação pelas características inerentes ao exercício da especialidade de MGF (I); vontade de experimentar MGF, enquanto aguardo por outra alternativa (E); ter sentido desde sempre vontade e desejo de ser Médico(a) de Família (I).

\begin{tabular}{|c|c|c|c|}
\hline Variável sócio - demográfica & & & $\%$ \\
\hline \multirow[t]{2}{*}{ Idade } & Média (DP) & $30,21(6,28)$ & - \\
\hline & Mediana & 28 & - \\
\hline \multirow[t]{2}{*}{ Género } & Feminino & 83 & 76,1 \\
\hline & Masculino & 26 & 23,9 \\
\hline \multirow[t]{3}{*}{ Grupo etário } & $\leq 25$ anos & 26 & 23,9 \\
\hline & $26-34$ anos & 66 & 60,6 \\
\hline & $\geq 35$ anos & 17 & 15,6 \\
\hline \multirow[t]{2}{*}{ Nacionalidade } & Portuguesa & 87 & 79,8 \\
\hline & Outra & 22 & 20,2 \\
\hline \multirow[t]{2}{*}{ Tempo de exercício de medicina } & Média (DP) & $3,04(3,87)$ & - \\
\hline & Mediana & 2 & - \\
\hline \multirow[t]{2}{*}{ Experiência anterior em Clínica Geral } & Sim & 14 & 12,8 \\
\hline & Não & 95 & 87,2 \\
\hline \multirow[t]{3}{*}{ Localização em relação ao internato } & Zona Norte & 43 & 39,4 \\
\hline & Zona Centro & 16 & 14,7 \\
\hline & Zona Sul & 50 & 45,9 \\
\hline \multirow[t]{2}{*}{ Escolha de MGF como opção inicial } & Sim & 86 & 78,9 \\
\hline & Não & 23 & 21,1 \\
\hline
\end{tabular}

$D P=$ desvio padrão
- Escala de avaliação utilizada: tratando-se de uma medida com a possibilidade de graduar a resposta aos itens em intervalos regulares, considerámos que elas obedecem, em termos métricos, às características das escalas intervalares desenvolvidas por Likert, que refere que os intervalos destas escalas devem ser iguais (rating scales).21-22 Desta forma, o tratamento dos itens que identificam os motivos de escolha da especialidade foi feito com base em análise quantitativa, somando os valores identificados pelos sujeitos, em cada item..$^{20-21}$

- Análise estatística:

Para além das estatísticas descritivas dos itens, utilizou-se análise bivariada no sentido de estudar as relações entre as variáveis, em função da caracterização da população, nomeadamente o Coeficiente de correlação Rho-de-Pearson, Teste de Mann-Whitney (quando comparadas duas amostras) e Teste de Kruskal-Wallis (quando comparadas três amostras), após verificação de todos os seus pressupostos.

Para a análise do grau de influência dos «Motivos de escolha da especialidade» de MGF, utilizou-se o valor da «moda», identificando o motivo mais frequentemente invocado pelos respondentes em cada item e calculou-se a percentagem de médicos que apontaram esse item como o mais influente nas suas escolhas.

Para a análise das dimensões que representam os Factores Intrínsecos e Extrínsecos, agruparam-se os itens identificados como intrínsecos e como extrínsecos, respectivamente. Utilizou-se o somatório das pontuações obtidas nos mesmos, para cada dimensão, e para além da análise descritiva, fez-se análise relacional em função da caracterização da população do estudo.

Garantida a confidencialidade das respostas obtidas no questionário, as mesmas foram codificadas e registadas numa base de dados, e o tratamento estatístico dos resultados foi processado no programa informático SPSS (versão 14,0), adoptando-se o nível de significância de 0,05.

\section{RESULTADOS}

Dos 228 médicos inquiridos, foram obtidos 109 questionários correctamente preenchidos (taxa de respos- 
ta $=47,8 \%$ ). A caracterização sócio-demográfica dos participantes no estudo, anteriormente reportada, ${ }^{18} \mathrm{e}$ resumida no Quadro I.

Analisados os «Motivos de Escolha da especialidade» de MGF (Quadro II), verifica-se que os itens que apresentavam valores médios mais próximos do seu valor máximo, correspondem a aspectos relacionados com factores identificados pela literatura como intrínsecos à especialidade, nomeadamente «Maior abrangência de áreas médicas abordadas» $(M=4,5 ; D P=0,79)$, «Gostar do contacto com pessoas» $(M=4,4 ; D P=0,76)$, «Gostar de ajudar pessoas doentes» $(M=4,4 ; D P=0,72) \mathrm{e}$ «Vocação pelas características inerentes ao exercício da especialidade de Medicina Geral e Familiar» ( $M=4,1 ; D P$ $=0,90$ ). Contrariamente, os itens onde se verifica menor pontuação são "Possibilidade de ter uma boa remuneração» $(M=2,0 ; D P=1,08)$, «Prestígio social da $M G F »(M=1,7 ; D P=1,01)$ e «Vontade de experimentar $M G F$, enquanto aguardo por outra alternativa» $(M=1,6$; $D P=1,09$ ).

A análise do grau de influência dos «Motivos de escolha da especialidade» de MGF (Figura 1) revela que «Gostar de ajudar pessoas doentes» (referidos por 50,5\% dos sujeitos), «Gostar do contacto com pessoas» (referidos por 55,0\% dos sujeitos) e «Maior abrangência das áreas médicas abordadas» (referidos por $57,8 \%$ dos sujeitos) foram os factores que maior proporção dos sujeitos valorizou como tendo muitíssima influência $(M o$ =5) na escolha da especialidade de Medicina Geral e Familiar.

A «Vocação pelas características inerentes ao exercício da especialidade de MGF» (referidos por $45,0 \%$ dos sujeitos) e a «Ter sentido desde sempre vontade e desejo de ser Médico de Família» (referidos por apenas 31,2\% dos sujeitos) foram apontados com a mesma frequência ( $M o$ =4) que factores relacionados com questões de ordem pessoal, como a «Disponibilidade para a família e outros interesses não profissionais» e o «Contacto anterior com o exercício de MGF».

Os motivos «Nota no exame de ingresso á especialidade» não tiveram qualquer influência $(M o=1)$, para mais de $45,0 \%$ dos respondentes, assim como a «Vontade de experimentar MGF, enquanto aguardo por outraalternativa» (Figura 1) assinalado por cerca de 71,8\% dos médicos como não tendo qualquer influência $(M o$ =1); este último motivo corresponde ao valor médio

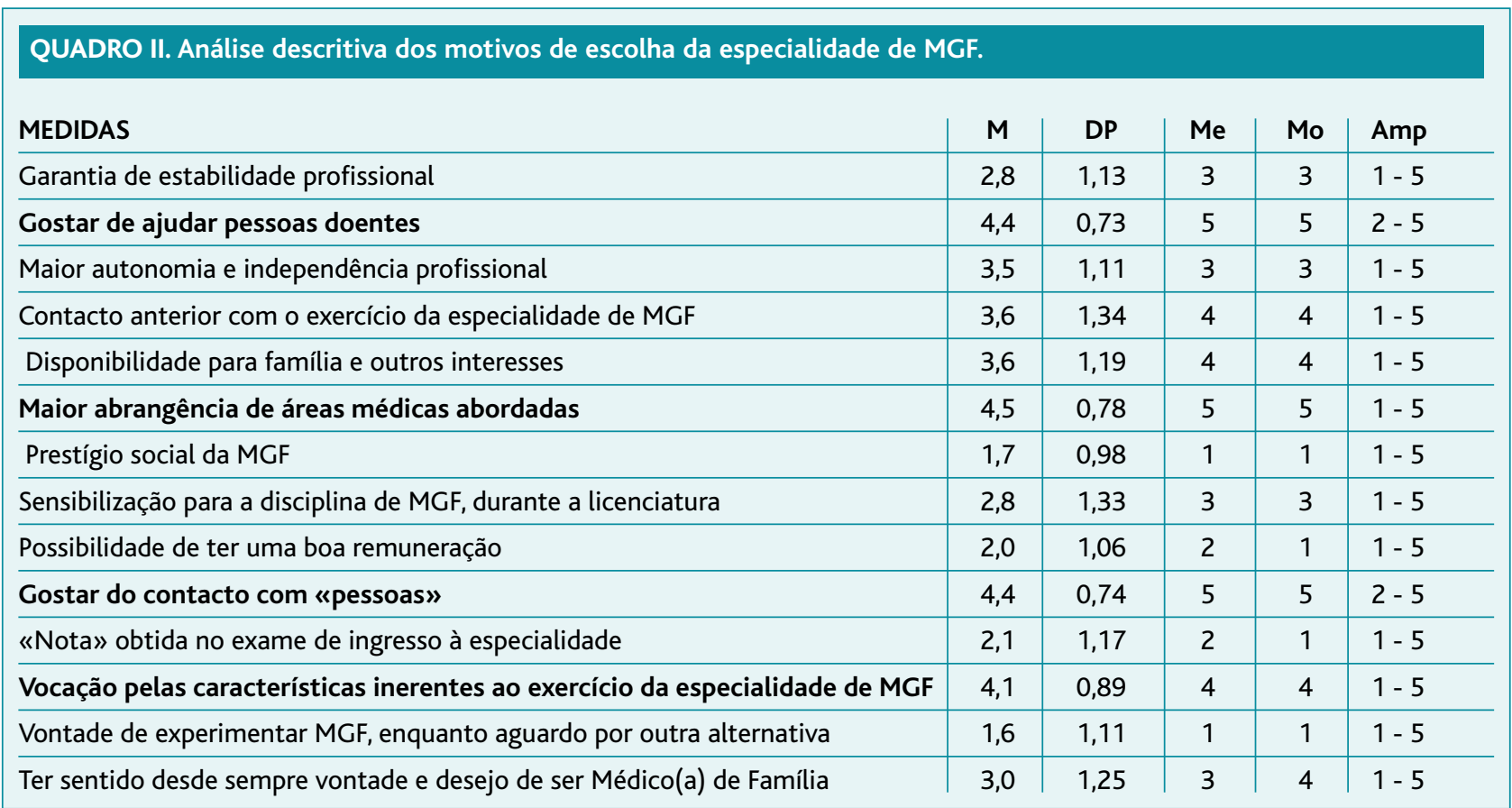

$M=$ média; $D P=$ desvio padrão; $M e=$ mediana; $M o=$ moda $; A m p=$ amplitude . 


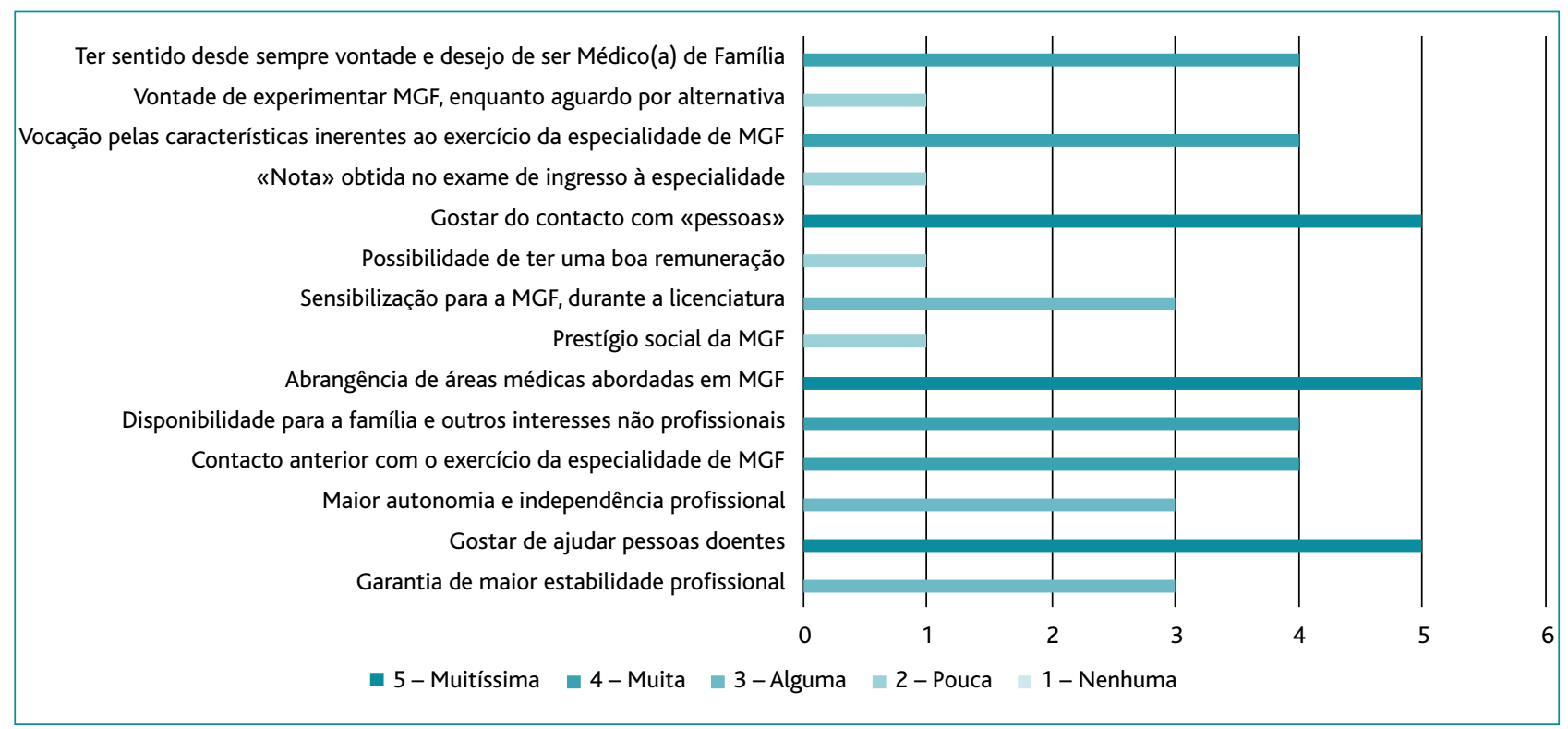

Figura 1. Representação gráfica da distribuição dos motivos de escolha da especialidade (valor da «moda»).

mais baixo dos motivos estudados $(M=1,6 ; D P=1,09)$. Por outro lado, o motivo «Ter uma boa remuneração» foi identificado como não tendo qualquer influência por $45,8 \%$ dos respondentes $(M o=1)$, embora seja referido como tendo "pouca» influência por $29,4 \%$ e «alguma» influência por $17,4 \%$ da amostra $(M=2,0 ; D P=1,08)$, a par de aspectos relacionados com o prestígio social da $\operatorname{MGF}(M=1,7 ; D P=1,0)$.

Conforme referido anteriormente $\mathrm{o}$ "Contacto anterior com o exercício da especialidade de MGF», ou seja, a experiência adquirida durante os estágios de MGF, anteriormente ao exame de acesso à especialidade (Quadro II e Figura 1), influenciou de forma consistente a escolha desta especialidade.

A análise da influência da escola médica na escolha da especialidade de Medicina Geral e Familiar, através do item «Sensibilização durante a licenciatura» (Figura 1) foi identificada por $23,4 \%$ da amostra deste estudo como tendo «alguma influência» $(M o=3)$, com uma distribuição uniforme nas respostas apontadas sobre essa influência $(M=2,8 ; D P=1,33)$.

Através da análise bivariada, para comparação de médias entre os motivos de escolha e a caracterização da amostra em estudo, verificam-se algumas diferenças estatisticamente significativas, na forma como os médicos identificam os seus motivos de escolha. São os médicos mais jovens os que valorizam mais a «Possibilidade de ter maior disponibilidade para a família e outros interesses» (Rho $=-0,22 ; p=0,03$ ) (Quadro III). Para os médicos internos do género masculino, aspectos como o "Prestígio social da MGF» (Dif. $M=13,8 ; p=$ 0,03 ) e a "Nota obtida no exame de acesso à especialidade» (Dif. $M=12,8 ; \mathrm{p}=0,04$ ) foram mais valorizados como influenciando as suas escolhas do que para o género feminino, contrariamente às mulheres que responderam valorizando mais o item «Ter sentido desde sempre a vontade e o desejo de ser Médico(a) de Família» (Dif. $M=13,9 ; p=0,03$ ) do que os homens (Quadro IV).

A forma como os dois grupos de médicos internos, portugueses e estrangeiros, valorizaram os diferentes motivos de escolha revela diferenças, com elevado significado estatístico, em relação a alguns motivos, sendo os estrangeiros os que apresentam as médias mais elevadas em itens identificados como extrínsecos às características da especialidade, como «Garantia de maior estabilidade profissional» (Dif. $M=17,6 ; p=0,03) e « V o n$ tade de experimentar enquanto aguardam por alternativas» (Dif. $M=15,1 ; p=0,02$ ) (Quadro V).

Entre o grupo de médicos internos que trabalhou anteriormente em Clínica Geral, de forma autónoma, num Centro de Saúde, e o que não o fez (Quadro VI), 


\begin{tabular}{|c|c|c|}
\hline Medidas & Rho & $\mathbf{P}$ \\
\hline Garantia de maior estabilidade profissional & 0,12 & 0,21 \\
\hline Gostar de ajudar pessoas doentes & $-0,04$ & 0,69 \\
\hline Maior autonomia e independência profissional & $-0,01$ & 0,89 \\
\hline Contacto anterior com o exercício da especialidade de MGF & 0,13 & 0,22 \\
\hline Possibilidade de ter maior disponibilidade para a família e outros interesses & $-0,22$ & 0,03 \\
\hline Especialidade caracterizada por uma grande abrangência das áreas abordadas & $-0,14$ & 0,54 \\
\hline Prestígio social da MGF & 0,06 & 0,58 \\
\hline Sensibilização para a MGF, durante a licenciatura & 0,02 & 0,81 \\
\hline Ter uma boa remuneração & $-0,12$ & 0,21 \\
\hline Gostar do «contacto» com pessoas & $-0,07$ & 0,45 \\
\hline «Nota» obtida no exame de ingresso à especialidade & $-0,02$ & 0,82 \\
\hline Vocação pelas características inerentes ao exercício da MGF & 0,03 & 0,76 \\
\hline Vontade de experimentar, enquanto aguardo por outras alternativas & $-0,16$ & 0,11 \\
\hline Ter sentido desde sempre a vontade e o desejo de ser Médico(a) de Família & 0,07 & 0,72 \\
\hline
\end{tabular}

Coeficiente de correlação de Rho de Spearman $(p<0,05)$. do Centro de Saúde (QuadroVIII), tendo sido os médicos internos da zona Norte os que valorizaram mais este motivo na influência da escolha de MGF (Mean Rank $=61,9$ ), em relação aos da zona Centro (Mean Rank $=44,0), \mathrm{e}$ igualmente da zona Sul (Mean Rank = 46,6).

A forma como o motivo «Sensibilização para a MGF durante a licenciatura» foi valorizado revela diferenças, con-

verificam-se diferenças, sendo os médicos internos que não passaram por esta experiência os que atribuíram maior valorização nas suas escolhas ao motivo «Possibilidade de ter maior disponibilidade para a família elou outros interesses não profissionais» (Dif. $M=17,8 ; p=$ $0,03)$.

Pela análise de comparação entre as zonas de localização dos centros de saúde, onde os médicos internos se encontram colocados e os motivos de escolha verificam-se diferenças com significado estatístico, para alguns dos itens analisados (Quadro VII). A valorização da «Maior autonomia e independência profissional» revela diferenças consoante a localização

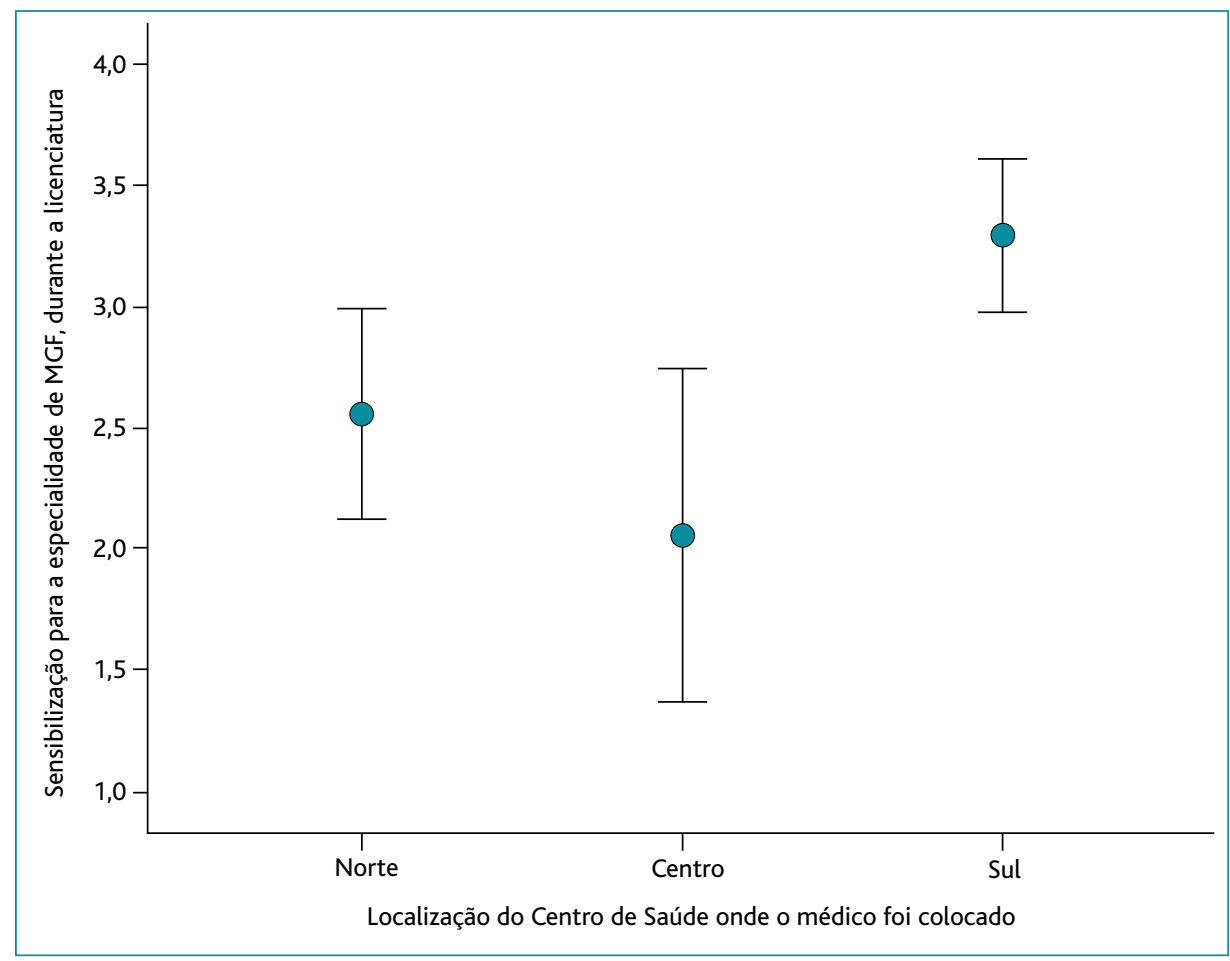

Figura 2. Representação gráfica da comparação do motivo «Sensibilização para a MGF, durante a licenciatura» segundo a localização do Centro de Saúde dos médicos internos (Teste Kruskal- Wallis). 


\begin{tabular}{|c|c|c|c|c|}
\hline Medidas & Género & $\mathbf{N}$ & Média dos Ranks & $\mathbf{P}$ \\
\hline \multirow[t]{2}{*}{ Garantia de maior estabilidade profissional } & Masc. & 26 & 54,5 & 0,6 \\
\hline & Fem. & 83 & 51,2 & \\
\hline \multirow[t]{2}{*}{ Gostar de ajudar pessoas doentes } & Masc. & 26 & 48,2 & 0,42 \\
\hline & Fem. & 83 & 53,2 & \\
\hline \multirow[t]{2}{*}{ Maior autonomia e independência profissional } & Masc. & 26 & 56,9 & 0,37 \\
\hline & Fem. & 83 & 50,6 & \\
\hline \multirow[t]{2}{*}{ Contacto anterior com o exercício da especialidade de MGF } & Masc. & 26 & 43,7 & 0,10 \\
\hline & Fem. & 83 & 54,7 & \\
\hline \multirow{2}{*}{$\begin{array}{l}\text { Possibilidade de ter maior disponibilidade para a família e outros } \\
\text { interesses }\end{array}$} & Masc. & 26 & 51,6 & 0,93 \\
\hline & Fem. & 83 & 52,1 & \\
\hline \multirow{2}{*}{$\begin{array}{l}\text { Ser uma especialidade caracterizada por uma grande abrangência } \\
\text { das áreas abordadas }\end{array}$} & Masc. & 26 & 50,2 & 0,69 \\
\hline & Fem. & 83 & 52,6 & \\
\hline \multirow[t]{2}{*}{ Prestígio social da MGF } & Masc. & 26 & 62,4 & 0,03 \\
\hline & Fem. & 83 & 48,7 & \\
\hline \multirow[t]{2}{*}{ Sensibilização para a MGF, durante a licenciatura } & Masc. & 26 & 48,2 & 0,46 \\
\hline & Fem. & 83 & 53,2 & \\
\hline \multirow[t]{2}{*}{ Ter uma boa remuneração } & Masc. & 26 & 51,2 & 0,87 \\
\hline & Fem. & 83 & 52,3 & \\
\hline \multirow[t]{2}{*}{ Gostar do «contacto» com pessoas } & Masc. & 26 & 44,6 & 0,11 \\
\hline & Fem. & 83 & 54,4 & \\
\hline \multirow[t]{2}{*}{ «Nota» obtida no exame de ingresso à especialidade } & Masc. & 26 & 61,7 & 0,04 \\
\hline & Fem. & 83 & 48,9 & \\
\hline \multirow[t]{2}{*}{ Vocação pelas características inerentes ao exercício da MGF } & Masc. & 26 & 48,1 & 0,42 \\
\hline & Fem. & 83 & 53,2 & \\
\hline \multirow{2}{*}{$\begin{array}{l}\text { Vontade de experimentar, enquanto aguardo por outras } \\
\text { alternativas }\end{array}$} & Masc. & 26 & 54,7 & 0,52 \\
\hline & Fem. & 83 & 51,1 & \\
\hline Ter sentido desde sempre a vontade e o & Masc. & 26 & 41,4 & 0,03 \\
\hline desejo de ser Médico(a) de Família & Fem. & 83 & 55,4 & \\
\hline
\end{tabular}

Teste de Mann Whitney $(p<0,05)$

soante a distribuição geográfica do Centro de Saúde onde se encontravam colocados (Quadro VII e Figura 2); os médicos da zona Sul valorizaram mais este motivo na influência das suas escolhas (Mean Rank $=62,3$ ), em relação aos da zona Norte (Mean Rank $=45,3$ ), e igualmente da zona Centro (Mean Rank $=35,8$ ). Identificando a faculdade de origem dos médicos internos que iniciaram o IMMGF na zona Sul, utilizando o Teste de Mann-Whitney, não se encontraram diferenças com significado estatístico, entre os médicos das duas escolas médicas maioritariamente em causa (Dif. $M=$
1,19), relativamente à forma como foi valorizado o motivo «Sensibilização para a especialidade de MGF, $d u$ rante a licenciatura» $(p=0,73)$.

Entre as dimensões identificadas como Factores Intrínsecos ou seja, intrinsecamente relacionados com as características da especialidade e Factores Extrínsecos, relacionados com questões de ordem pessoal, social, e profissional (Figura 3), verificou-se uma diferença entre as suas médias (Dif. $M=13,7$ ), sendo os Factores Intrínsecos $(M=30,4 \pm 4,37$; Max. - Min. = 25) mais valorizados pelos médicos deste estudo do que os Factores 


\begin{tabular}{|c|c|c|c|c|}
\hline Medidas & Nacionalidade & $\mathbf{N}$ & Média dos Ranks & $\mathbf{P}$ \\
\hline Garantia de maior estabilidade profissional & $\begin{array}{l}\text { Portuguesa } \\
\text { Outra }\end{array}$ & $\begin{array}{l}87 \\
22\end{array}$ & $\begin{array}{l}45,1 \\
62,6\end{array}$ & 0,03 \\
\hline Gostar de ajudar pessoas doentes & $\begin{array}{l}\text { Portuguesa } \\
\text { Outra }\end{array}$ & $\begin{array}{l}87 \\
22\end{array}$ & $\begin{array}{l}48,8 \\
39,4\end{array}$ & 0,19 \\
\hline Maior autonomia e independência profissional & $\begin{array}{l}\text { Portuguesa } \\
\text { Outra }\end{array}$ & $\begin{array}{l}87 \\
22\end{array}$ & $\begin{array}{l}47,3 \\
48,6\end{array}$ & 0,87 \\
\hline Contacto anterior com o exercício da especialidade de MGF & $\begin{array}{l}\text { Portuguesa } \\
\text { Outra }\end{array}$ & $\begin{array}{l}87 \\
22\end{array}$ & $\begin{array}{l}49,0 \\
37,9\end{array}$ & 0,15 \\
\hline $\begin{array}{l}\text { Possibilidade de ter maior disponibilidade para a família e } \\
\text { outros interesses }\end{array}$ & $\begin{array}{l}\text { Portuguesa } \\
\text { Outra }\end{array}$ & $\begin{array}{l}87 \\
22\end{array}$ & $\begin{array}{l}48,7 \\
40,0\end{array}$ & 0,27 \\
\hline $\begin{array}{l}\text { Ser uma especialidade caracterizada por uma grande } \\
\text { abrangência das áreas abordadas }\end{array}$ & $\begin{array}{l}\text { Portuguesa } \\
\text { Outra }\end{array}$ & $\begin{array}{l}87 \\
22 \\
\end{array}$ & $\begin{array}{l}46,6 \\
53,1\end{array}$ & 0,36 \\
\hline Prestígio social da MGF & $\begin{array}{l}\text { Portuguesa } \\
\text { Outra }\end{array}$ & $\begin{array}{l}87 \\
22\end{array}$ & $\begin{array}{l}47,6 \\
46,8\end{array}$ & 0,90 \\
\hline Sensibilização para a MGF, durante a licenciatura & $\begin{array}{l}\text { Portuguesa } \\
\text { Outra }\end{array}$ & $\begin{array}{l}87 \\
22\end{array}$ & $\begin{array}{l}46,8 \\
51,8\end{array}$ & 0,53 \\
\hline Ter uma boa remuneração & $\begin{array}{l}\text { Portuguesa } \\
\text { Outra }\end{array}$ & $\begin{array}{l}87 \\
22\end{array}$ & $\begin{array}{l}46,2 \\
55,5\end{array}$ & 0,22 \\
\hline Gostar do «contacto com pessoas & $\begin{array}{l}\text { Portuguesa } \\
\text { Outra }\end{array}$ & $\begin{array}{l}87 \\
22\end{array}$ & $\begin{array}{l}47,0 \\
50,6\end{array}$ & 0,60 \\
\hline «Nota» obtida no exame de ingresso à especialidade & $\begin{array}{l}\text { Portuguesa } \\
\text { Outra }\end{array}$ & $\begin{array}{l}87 \\
22\end{array}$ & $\begin{array}{l}46,5 \\
63,8\end{array}$ & 0,04 \\
\hline Vocação pelas características inerentes ao exercício da MGF & $\begin{array}{l}\text { Portuguesa } \\
\text { Outra }\end{array}$ & $\begin{array}{l}87 \\
22\end{array}$ & $\begin{array}{l}46,5 \\
53,5\end{array}$ & 0,35 \\
\hline $\begin{array}{l}\text { Vontade de experimentar enquanto aguardo por outras } \\
\text { alternativas }\end{array}$ & $\begin{array}{l}\text { Portuguesa } \\
\text { Outra }\end{array}$ & $\begin{array}{l}87 \\
22\end{array}$ & $\begin{array}{l}45,4 \\
60,5\end{array}$ & 0,02 \\
\hline $\begin{array}{l}\text { Ter sentido desde sempre a vontade e o } \\
\text { desejo de ser Médico(a) de Família }\end{array}$ & $\begin{array}{l}\text { Portuguesa } \\
\text { Outra }\end{array}$ & $\begin{array}{l}87 \\
22\end{array}$ & $\begin{array}{l}47,9 \\
45,2\end{array}$ & 0,74 \\
\hline
\end{tabular}

Teste de Mann Whitney $(p<0,05)$

Extrínsecos $(M=15,7 \pm 4,37$; Max. - Min. = 24).

A forma como esta amostra atribuiu relevância a Factores Intrínsecos ou a Factores Extrínsecos na opção pela especialidade de MGF não revela estar relacionada com a sua caracterização sócio-demográfica e profissional, excepto para a distribuição por género.

Entre os homens e as mulheres, existem diferenças com significado estatístico em relação à escolha de MGF ( $p=0,03$ ) influenciada por Factores Intrínsecos, tendo sido as mulheres (Dif. $M=4,9$ ) a atribuir mais im- portância a estes motivos (Mean Rank $=55,6$ ) em relação aos homens (Mean Rank=40,7).

Quanto à distribuição etária desta amostra, não existe correlação com significado estatístico, quer com os Factores Intrínsecos ( $r h o=0,07 ; p=0,47)$, quer com os Factores Extrínsecos ( $r h o=-0,11 ; p=0,37)$. Em relação à nacionalidade dos médicos, a mesma análise não revela a existência de diferenças estatisticamente significativas na forma como são valorizados os Factores Intrínsecos $(p=0,74)$ e Extrínsecos $(p=0,70)$. 
Não existe igualmente relação, com significado estatístico, entre a experiência relativa ao trabalho anterior, de forma autónoma, num Centro de Saúde, na escolha de MGF preferencialmente por Factores Intrínsecos $(p=0,41)$ ou Extrínsecos $(p=0,50)$.

A variabilidade entre as diferentes zonas de localização do Centro de Saúde revela que as médias das ordenações das respostas são iguais entre as zonas, quer para os Factores Intrínsecos $(p=0,08)$ - Norte $(M=48,7)$, Centro $(M=39,9)$ e Sul $(M=58,30)$, quer para os Facto- res Extrínsecos $(p=0,33)$ - Norte $(M=57,2)$, Centro $(M$ $=53,5)$ e Sul $(M=47,7)$.

\section{DISCUSSÃO}

A redução do número de efectivos médicos ao nível da MGF nos últimos anos e a necessidade de esclarecer as razões que levam os médicos a escolher esta especialidade justifica a pertinência desta investigação. No sentido de perceber não apenas a realidade actual em Por-

\begin{tabular}{|c|c|c|c|c|}
\hline Medidas & $\begin{array}{l}\text { Trabalho anterior } \\
\text { em Clínica Geral }\end{array}$ & $\mathbf{N}$ & $\begin{array}{l}\text { Média dos } \\
\text { Ranks }\end{array}$ & $\mathbf{P}$ \\
\hline Garantia de maior estabilidade profissional & $\begin{array}{l}\text { Sim } \\
\text { Não }\end{array}$ & $\begin{array}{l}14 \\
95\end{array}$ & $\begin{array}{l}61,8 \\
49,9\end{array}$ & 0,14 \\
\hline Gostar de ajudar pessoas doentes & $\begin{array}{l}\text { Sim } \\
\text { Não }\end{array}$ & $\begin{array}{l}14 \\
95\end{array}$ & $\begin{array}{l}54,4 \\
51,0\end{array}$ & 0,67 \\
\hline Maior autonomia e independência profissional & $\begin{array}{l}\text { Sim } \\
\text { Não }\end{array}$ & $\begin{array}{l}14 \\
95\end{array}$ & $\begin{array}{l}47,2 \\
52,2\end{array}$ & 0,54 \\
\hline Contacto anterior com o exercício da especialidade de MGF & $\begin{array}{l}\text { Sim } \\
\text { Não }\end{array}$ & $\begin{array}{l}14 \\
95\end{array}$ & $\begin{array}{l}61,6 \\
49,9\end{array}$ & 0,15 \\
\hline $\begin{array}{l}\text { Possibilidade de ter maior disponibilidade para a família } \\
\text { e outros interesses }\end{array}$ & $\begin{array}{l}\text { Sim } \\
\text { Não }\end{array}$ & $\begin{array}{l}14 \\
95\end{array}$ & $\begin{array}{l}36,2 \\
54,0\end{array}$ & 0,03 \\
\hline $\begin{array}{l}\text { Ser uma especialidade caracterizada por uma grande } \\
\text { abrangência das áreas abordadas }\end{array}$ & $\begin{array}{l}\text { Sim } \\
\text { Não }\end{array}$ & $\begin{array}{l}14 \\
95\end{array}$ & $\begin{array}{l}56,0 \\
50,8\end{array}$ & 0,48 \\
\hline Prestígio social da MGF & $\begin{array}{l}\text { Sim } \\
\text { Não }\end{array}$ & $\begin{array}{l}14 \\
95\end{array}$ & $\begin{array}{l}53,0 \\
51,3\end{array}$ & 0,83 \\
\hline Sensibilização para a MGF, durante a licenciatura & $\begin{array}{l}\text { Sim } \\
\text { Não }\end{array}$ & $\begin{array}{l}14 \\
95\end{array}$ & $\begin{array}{l}57,0 \\
50,6\end{array}$ & 0,44 \\
\hline Ter uma boa remuneração & $\begin{array}{l}\text { Sim } \\
\text { Não }\end{array}$ & $\begin{array}{l}14 \\
95\end{array}$ & $\begin{array}{l}49,0 \\
51,9\end{array}$ & 0,72 \\
\hline Gostar do «contacto» com pessoas & $\begin{array}{l}\text { Sim } \\
\text { Não }\end{array}$ & $\begin{array}{l}14 \\
95\end{array}$ & $\begin{array}{l}49,1 \\
51,9\end{array}$ & 0,72 \\
\hline «Nota» obtida no exame de ingresso à especialidade & $\begin{array}{l}\text { Sim } \\
\text { Não }\end{array}$ & $\begin{array}{l}14 \\
95\end{array}$ & $\begin{array}{l}49,0 \\
51,9\end{array}$ & 0,71 \\
\hline Vocação pelas características inerentes ao exercício da MGF & $\begin{array}{l}\text { Sim } \\
\text { Não }\end{array}$ & $\begin{array}{l}14 \\
95\end{array}$ & $\begin{array}{l}53,1 \\
51,2\end{array}$ & 0,81 \\
\hline $\begin{array}{l}\text { Vontade de experimentar enquanto aguardo por outras } \\
\text { alternativas }\end{array}$ & $\begin{array}{l}\text { Sim } \\
\text { Não }\end{array}$ & $\begin{array}{l}14 \\
95\end{array}$ & $\begin{array}{l}44,9 \\
52,6\end{array}$ & 0,27 \\
\hline $\begin{array}{l}\text { Ter sentido desde sempre a vontade e o } \\
\text { desejo de ser Médico(a) de Família }\end{array}$ & $\begin{array}{l}\text { Sim } \\
\text { Não }\end{array}$ & $\begin{array}{l}14 \\
95\end{array}$ & $\begin{array}{l}56,1 \\
50,8\end{array}$ & 0,52 \\
\hline
\end{tabular}




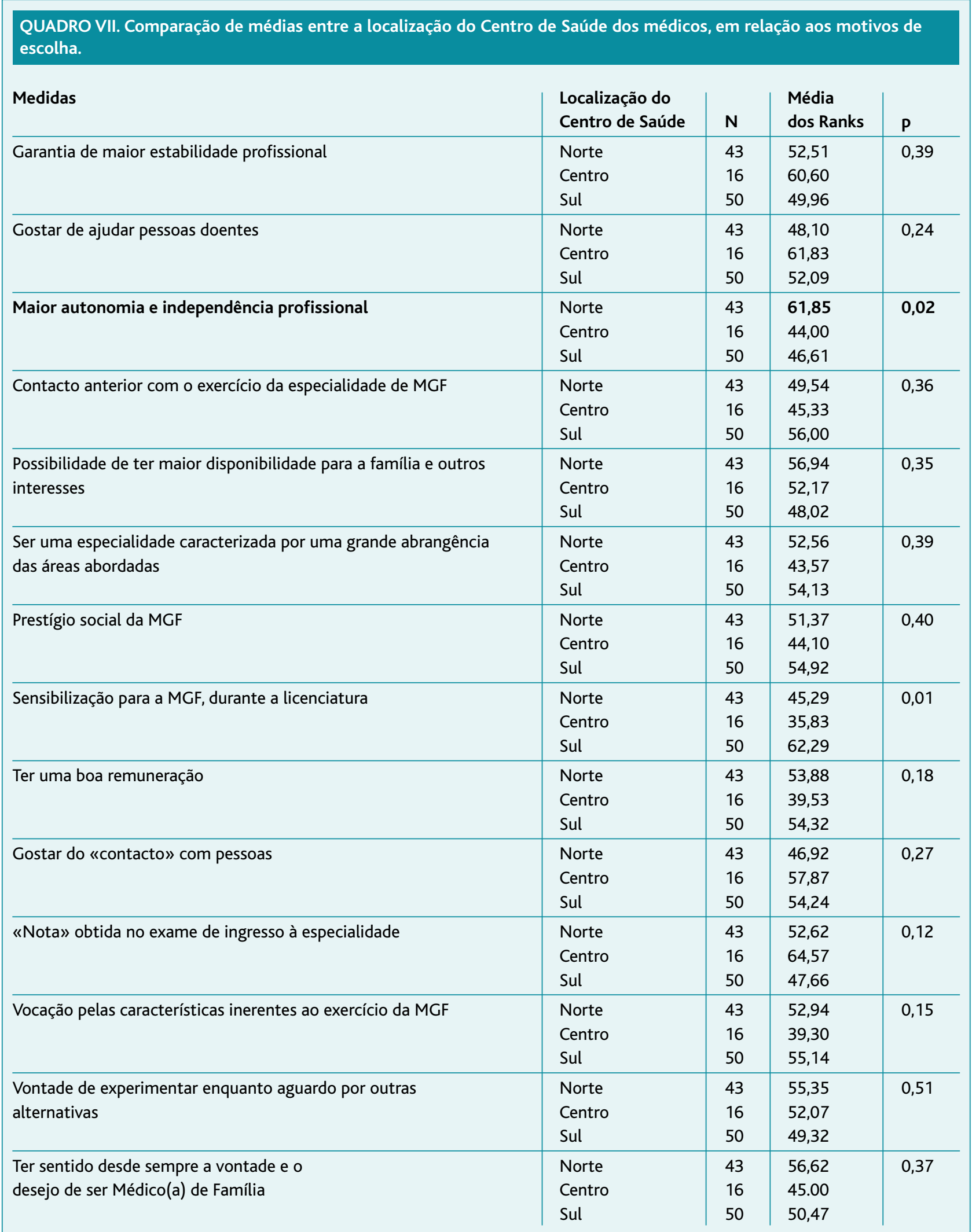

Teste de Kruskal-Wallis $(\mathrm{p}<0,05)$ 


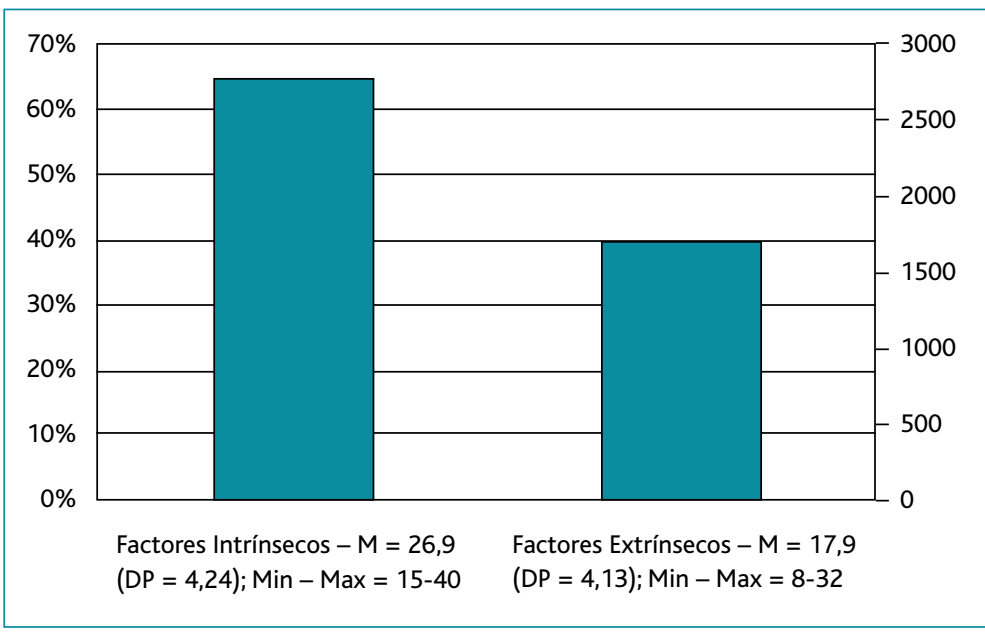

Figura 3. Representação gráfica da análise descritiva dos factores intrínsecos e extrínsecos.

tugal, parece-nos que, para além de consolidar informação já divulgada anteriormente ${ }^{11,18,23}$ o estudo permitiu identificar aspectos que podem contribuir para aprofundar e esclarecer uma problemática que continua a despertar opiniões controversas.

Os resultados apresentados anteriormente confirmam que o perfil do médico interno neste país se manteve muito semelhante ao longo destes últimos anos. ${ }^{11,18,23} \mathrm{~A}$ percentagem mais elevada de médicos a referir optar pela especialidade de MGF como a sua primeira opção ${ }^{18}$ revelou uma inversão do padrão de escolha anteriormente comentado na literatura. ${ }^{9}$

No grupo de médicos em análise, sendo a escolha de MGF congruente com a opção inicialmente pensada para o seu futuro profissional, as respostas obtidas aos itens analisados na presente investigação revelam que essa opção se faz por motivações relacionadas com as características da especialidade e inerentes ao exercício da mesma.

A classificação no exame de acesso à especialidade foi referida neste estudo como não tendo qualquer influência nas escolhas por cerca de metade dos médicos, pelo que este motivo parece não ter sido um determinante para a evolução positiva deste padrão de escolha, contrariamente ao expectável face à descrição da ocupação maciça das vagas de MGF por médicos com as classificações mais baixas. ${ }^{9}$ Pensamos que a classificação mais baixa no exame de acesso à especialidade poderá não significar necessariamente baixa motivação para a MGF, pois que quem concorre sabe antecipadamente que a dificuldade na ocupação de vagas nesta especialidade não será um problema, e como tal, não estará necessariamente demasiado preocupado com «nota» que poderá ter nesse exame.

A influência de outros aspectos extrínsecos às características da especialidade, nomeadamente a possibilidade de uma boa remuneração ou a hipótese de «experimentar» para posteriormente tentar outra alternativa, foram dos motivos que menos contribuíram para a escolha de MGF neste grupo de médicos.

Contrariamente, os resultados revelam maior relevância dos Factores Intrínsecos, em que o interesse humanístico, identificado através dos itens «Gostar de ajudar pessoas doentes», «Gostar do contacto com pessoas» e «Ter sentido desde sempre a vontade o o desejo de ser Médico(a) de Família», teve bastante influência nas escolhas profissionais dos médicos internos de MGF deste estudo. Para além do seu elevado número, salienta-se que foram as mulheres que mais valorizaram razões intrínsecas à especialidade de MGF, nesta amostra, o que está de acordo com resultados publicados, em que as mulheres escolhem habitualmente especialidades em que é maior a componente relacional. ${ }^{13}$

O grau de influência identificado para cada um dos motivos de escolha analisados revela-nos que a maior abrangência de áreas médicas abordadas (especialidade generalista), o «gosto» pela relação interpessoal (medicina centrada na pessoa, na globalidade e continuidade de cuidados) e o gostar de ajudar pessoas «doentes» (importância da relação de ajuda) foram factores identificados como tendo tido «muitíssima» influência na escolha de MGF, o que corresponde aos aspectos descritos na literatura como os motivos que mais frequentemente são determinantes da escolha de MGF. ${ }^{12,14,17,19}$

Do mesmo modo, parece haver uma identificação entre os motivos mais frequentemente apontados para escolher MGF e os princípios em que se fundamenta esta especialidade. ${ }^{11} \mathrm{~A}$ "Vocação pelas características inerentes ao exercício da especialidade de MGF» assim como a «Maior abrangência de áreas médicas aborda- 
das» apontam para uma opção fundamentada num conhecimento prévio da MGF para cerca de $45 \%$ e $31 \%$ dos sujeitos, respectivamente. Assim, podemos afirmar que, mesmo que os médicos que iniciam o internato de MGF pudessem não estar interessados nesta especialidade na fase inicial do internato, respondem identificando-se com as características que mais distingue a MGF das outras especialidades. Questionamo-nos acerca da forma como evoluirá a motivação inicial para a MGF deste grupo de médicos, durante um programa de formação no IMMGF, em contexto real de trabalho, com o apoio de um Orientador de formação. Justificase o desenho de uma investigação no sentido de esclarecer qual o contributo que o mesmo poderá proporcionar, influenciando a motivação destes médicos e favorecendo a qualidade do exercício da MGF com consequente sensibilização de futuros jovens médicos.

Relativamente à análise do item «Contacto anterior com a especialidade de MGF» confirma-se que a influência de experiências profissionais anteriores, nomeadamente a experiência em estágios de MGF no contexto da licenciatura, assim como durante o Internato Geral, parecem ter contribuído para orientar as escolhas destes médicos, de acordo com dados descritos na literatura. ${ }^{14,19}$ Experiencias negativas anteriores nos estágios em Centros de Saúde são apontados como factores que afastam os médicos da MGF.9

Por outro lado, para os médicos que nunca trabalharam anteriormente de forma autónoma, num Centro de Saúde, quando optam pela MGF valorizam mais o item «Possibilidade de ter maior disponibilidade para a família e/ou outros interesses não profissionais» do que os que tiveram essa experiencia, parecendo transparecer uma percepção irrealista das condições de exercício da especialidade pelos médicos que iniciam o IMMGF.

$\mathrm{Na}$ análise crítica desta investigação, para além do carácter subjectivo da temática analisada, eventualmente influenciando as respostas, torna-se pertinente ter em conta o efeito da desejabilidade social, que não foi excluído no desenho do estudo, em particular pelo efeito resultante da identificação dos participantes.

Poder-se-ia considerar a necessidade de terem sido incluídos no presente estudo outros aspectos eventualmente invocados como motivos de escolha, nomeadamente os motivos identificados num instru- mento utilizado anteriormente. ${ }^{11}$ Atendendo à utilização de uma população de médicos no início do seu internato, interessava-nos o carácter exploratório do estudo, pelo que optámos pela linha metodológica atrás descrita.

Por outro lado, o instrumento utilizado e elaborado para esta investigação, foi alvo de uma metodologia de validação do conteúdo dos seus itens, através de validação facial, mas não foram estudadas as suas propriedades psicométricas, por não ser esse o objectivo deste trabalho, pelo que não pode ser considerada a fiabilidade necessária ao rigor da construção de uma escala de medida, em futuras investigações. A análise comparativa com resultados de outras investigações poderá contribuir para a validade externa das conclusões apresentadas neste trabalho.

Tendo como finalidade analisar a problemática das escolhas profissionais dos médicos que optam pela especialidade de MGF, no início da sua formação em internato médico, e pensando ter atingido os objectivos deste estudo, consideramos a necessidade de mais investigação, em especial dirigida aos médicos que não optam pela MGF, no sentido de saber as razões que afastam os médicos de especialidade de $\mathrm{MGF}^{17,24,25} \mathrm{em}$ Portugal como em outros países.

Em síntese, os resultados permitem afirmar que, para o grupo profissional estudado, a opção pela MGF se faz maioritariamente por razões intrinsecamente relacionadas com a especialidade, concluindo igualmente que os médicos «vêm para esta especialidade porque gostam do que nela se faz e do que ela representa». ${ }^{11}$

No entanto, a influência das escolas médicas no percurso profissional dos médicos através da análise da variável «Sensibilização durante a licenciatura» foi pouco expressiva nesta investigação, sendo importante perceber as razões deste comportamento, através de investigação mais alargada. Mais relevante do que estudar essas razões nos médicos em fase pós-graduada seria igualmente importante dirigir a investigação aos estudantes de medicina, no sentido de perceber quais as suas intenções de carreira, no início e final da licenciatura, assim como a influência quer dos currículos quer dos professores e tutores de MGF nessa evolução.

A atracção pelas sub-especialidades hospitalares é uma realidade que tem repercussões sobre as escolhas profissionais dos jovens médicos na actualidade, e que 
faz sentir a sua influência dentro das faculdades. . $^{2,414,15,19}$ O ensino pré-graduado, essencialmente centrado no hospital, não parece ter contribuído para melhorar de forma considerável a preferência inicial por esta especialidade, no presente estudo. Para os jovens médicos, a MGF aparenta continuar a ser percepcionada como uma especialidade de alternativa, de baixo conteúdo intelectual e pouco atractiva, numa cultura médica tradicionalmente hospitalocêntrica, que urge ultrapassar. ${ }^{14,24,26}$ Considera-se a necessidade da reestruturação do ensino médico, incluindo o papel da disciplina de MGF na melhoria da percepção dos alunos de medicina acerca desta especialidade e da melhor compreensão da realidade que afasta os jovens licenciados da MGF.

Concluindo, a evolução positiva do padrão de escolha ${ }^{18} \mathrm{e}$ a opção pela MGF maioritariamente centrada em motivações intrinsecamente relacionadas com a especialidade sugere que actualmente a Medicina Geral e Familiar aparenta ser mais do que uma «alternativa de recurso" para os médicos que iniciam o IMMGF em Portugal.

A necessidade de aumentar o número de efectivos médicos em exercício, no âmbito da Medicina Geral e Familiar, actualmente em Portugal, é uma realidade que requer a adopção de todas estratégias possíveis, incluindo as dirigidas ao ensino médico pré-graduado, ${ }^{9}$ no sentido de aumentar a atractividade desta especialidade.

\section{AGRADECIMENTOS}

Ao Professor Doutor Saul Neves de Jesus e Professor Doutor José Pestana Cruz, da Faculdade de Ciências Humanas e Sociais da Universidade do Algarve, pelo contributo no trabalho de validação do conteúdo dos itens do instrumento utilizado.

\section{REFERENCIAS BIBLIOGRÁFICAS}

1. Meads G. Primary Care in the twenty-first century. An internacional perspective. United Kingdom : Radcliff Publishing; 2006.

2. Dwinnell $B$, Lorraine $A$. Why we are on the cusp of a generalist crisis. Acad Med 2001 Jul; 76 (7): 707 - 2.

3. Henderson E, Berlin A, Fuller J. Attitude of medical students towards general practice and general practitioners. Br J Gen Practice 2002 Mai; 52: 359 - 363.

4. Rosser $\mathrm{W}$. The decline of family medicine as a career choice [Acedido em 25/4/2009] Disponível em: http://www.cmaj.ca/cgi/content/ full/166/11/1419

5. Hays RB. Choosing a career in general practice: the influence of medical schools. Med Educ 1993 May; 27 (3): 254 - 8.
6. Tandeter H, Granek-Catarivas M. Choosing Primary Care? Influences of medical school curricula on career pathways. IMAJ 2001; 3: 969 - 972.

7. Sandholzer H. Is primary care a lost cause? CMAJ 2007 22;176(11):1570 $-1$.

8. Bragança de Sá E. Porquê escolher a especialidade de Clinica Geral? Post Grad Medicine (Editorial) 2005 Jan; 23 (1).

9. Sequeira Carlos J. Internato de MGF - vagas de crise $\left(2^{\circ}\right.$ episódio). Jornal Médico de Família 2005 Jan; 80: 36 - 2.

10. Brito de Sá A. A apologia das aptidões (Editorial). Rev Port Clin Geral 2003; 19: 541 -2.

11. Castro H. Motivos invocados para a escolha de Clinica Geral. Rev Port Clin Geral 2000; 16: 427 - 39.

12. Soethout MBM, ten Cate TJ, van der Val G. Factors associated with the nature, timing and stability of the specialty career choices of recently graduated doctors in european countries. http://www.med-ed-online.org [Online] 2004.

13. McFarland KF, Rhoades DR. Gender-related values and medical specialty choice. Academic Psychiatry 1998 Dec; 22: 236-239.

14. Senf JH, Campos-Outcalt D, Kutob R. Factors related to the choice of family medicine: a reassesment and literature review. J Am Board Family Pract 2003 Nov; 16(6): 502-512.

15. Griffith $\mathrm{CH}$, Georgesen JC, Wilson F. Specialty choices of students who actually have choices. the influence of excellent clinical teachers. Acad Med 2000; 75: 278-82.

16. Jordan J, Brown JB, Russel G. Choosing family medicine. What influence medical students? Can Fam Physician 2003; 49:1131-7.

17. Schafer $S$, et al. Rejecting Family Practice:Why medical students switch to other specialties. Fam Med 2000; 32(5): 320-5.

18. Gaspar D. Internato Médico de Medicina Geral e Familiar - Quem Somos? O perfil do Médico Interno de Medicina Geral e Familiar, em Portugal. Acta Med Port 2010;23:39-50.

19. Wright $B$, et al. Career choice of new medical studentes at three canadian universities: family medicine versus specialty medicine. CMAJ 2004 Jun; 170(13):1920-4.

20. Almeida LS, Freire T. Metodologia de investigação em Psicologia e Educação.(3 ${ }^{\mathrm{a}}$ ed). Braga: Psiquilíbrios; 2003.

21. Hill MM, Hill A. A investigação por questionário ( $2^{a}$ ed) Lisboa: Edições Sílabo; 2002.

22. Pestana MH, Gageiro JN. Análise de dados para ciências sociais. a complementaridade do SPSS (4ª ed) Lisboa: Edições Sílabo; 2005.

23. Outeirinho C. Internato Complementar de Clínica Geral na Zona Norte - Uma década. Rev Port Clin Geral 2005: 21; 69-78.

24. Burack JH et al. A study of medical students 'speacialty choice pathways: trying on possible selves. Acad Med 1997 Jun; 72(6): 534-41.

25. Colwill JM. Where have all the primary care aplicants gone? N Eng J Med 1992 Jun 26; 326 (6): 387-393.

Os autores declararam não possuir conflitos de interesses.

\section{ENDEREÇO PARA CORRESPONDÊNCIA}

Dina Gaspar

Urbanização Horta das Figuras Lote 31 - Bloco E, $5^{\circ}$ Dto.

8005 - 328 Faro

dina.gaspar@gmail.com 


\section{ABSTRACT}

\section{COOSING FAMILY MEDICINE: AN ALTERNATIVE OR FIRST OPTION?}

Objectives: To evaluate the influence of reasons presented by Family Medicine specialty residents, for the choice of this specialty, in Portugal; to analyze the importance of intrinsic motives related to Family Medicine, in their professional choice.

Study Design: Observational, cross-sectional, analytic study.

Setting: Mainland Portugal.

Participants: Doctors who began Family Medicine residency in Portugal, in $2005(\mathrm{~N}=228)$.

Methods: Postal questionnaire survey using 14 five points Likert scale items, representing the reasons identified as motives for choosing Family Medicine. Descriptive statistics were calculated and analytic tests were used to compare the relationship between the variables $(\alpha=0.05)$.

Results: The response rate was $47.8 \%(n=109)$. Factors intrinsically related to the characteristics of the specialty $(M=26.9$; $S D=4.24)$ were more relevant than the personal or others motives $(M=17.4 ; S D=4.13)$, in their option for Family Medicine; pregraduate training is referred as having some influence by $23.4 \%$ of the residents.

Conclusions: For most of Family Medicine residents the specialty choice is more intrinsically related with the characteristics of Family Medicine. We consider the need of rethinking the role of Family Medicine discipline in pregraduate curricula and the influence of the medical schools in specialty choice.

Keywords: Family Medicine; Medical Residency; Career Choice; Medical School Curricula.

\section{ERRATA}

No último número da Revista Portuguesa de Clínica Geral não foi incluída na lista de revisores de 2009 a Dra. Cristina Valadas (Endocrinologista). Pelo lapso, apresentamos as nossas desculpas. 